\title{
Specific Absorption Rate Assessment of Multiple Microstrip Patch Antenna Array
}

\author{
Nur Ilham Aliyaa Ishak ${ }^{1}$, Norhudah Seman ${ }^{{ }^{2}}$, Noor Asmawati Samsuri ${ }^{3}$ \\ Wireless Communivation Center (WCC), Faculty of Electrical Engineering, Universiti Teknologi Malaysia \\ *Corresponding author, e-mail: aliyaa_ishak@yahoo.com ${ }^{1}$, huda@fke.utm.my ${ }^{2}$, asmawati@fke.utm.my ${ }^{3}$
}

\begin{abstract}
Interaction between electromagnetic field (EMF) radiated from multiple antennas and human body is crucial to be explored as multiple antennas are the essential implemented devices to achieve the requirements of the future evolved fitth generation (5G) technology. Thus, this article presents a significant study of the radiated EMF effect from a single, and multiple antennas towards human through the assessment of specific absorption rate (SAR). The single antenna, $1 \times 2,1 \times 3$ and $1 \times 4$ arrays of microstrip patch antennas are designed to cover mobile operating frequencies of 0.8, 0.85, 0.9, 1.8, 2.1 and $2.6 \mathrm{GHz}$. Two types of human head phantoms are implemented in this study, which are specific anthropomorphic mannequin (SAM) and Voxel head model that placed close to single antenna or antenna array to investigate the penetration of EMF towards the human tissue. The single antenna or antenna array is placed with fixed distance of $10 \mathrm{~mm}$ from the phantom, which excited by maximum allowable power of $19 \mathrm{dBm}$ in CST Microwave Studio 2016. The effect of the radiated EMF that quantified by SAR parameter, which depicts satisfying results against the established standard limits at averaged $1 \mathrm{~g}$ and $10 \mathrm{~g}$ mass of tissues for all designated frequencies that utilized for single and multiple antennas.
\end{abstract}

Keywords: Specific absorption rate, Multiple antennas, Microstrip patch antenna, Electromagnetic field

Copyright $@ 2018$ Universitas Ahmad Dahlan. All rights reserved.

\section{Introduction}

The new challenge of mobile technology is to realize the 5th Generation (5G) goals in efficiently utilizing the existing and expanded bandwidth (BW) and shifting to higher frequency to provide adequate coverage and more bits/ $\mathrm{Hz}$. In addition, 5G aims in enabling device-to-device communication and interconnecting all wireless heterogonous networks to offer the seamless and consistent communication experience to users [1]. This new generation of mobile technology is expected to be deployed between 2020 and 2030 with 1000 times system capacity, 10 times spectral efficiency and energy efficiency, 25 times average call throughput [2], [3] and five times reduced end-to-end latency [4] for higher data rates, network capacity and advanced speed beyonds the current 4th Generation of Long Term-Evolution (4G LTE). Eventually as this new $5 \mathrm{G}$ technology not limited to human communication, but also device interactions, which involving the machine-to-machine and vehicle-to-vehicle communication, consequently the enormous increment of wireless devices that integrated to global wireless network by "Internet of Things" (IoT) that allows them to be functionally reachable and accessable. As to cope to the 5G's requirements, these devices must consist of multiple antennas. These antennas seamlessly emit the electromagnetic field (EMF) radiation in the form of non-ionizing radiation (NIR) [5], which can cause the thermal effect to human body. Thus, the interaction relations between the EMF emission from these multiple antennas and human tissue is significant to be studied [6], [7].

The rate of absorption energy from the EMF radiation by human body, which causes thermal effect can be computed by a dosimetric measurement known as specific absorption rate (SAR). SAR is the dosimetric measure of the rate at which energy is absorbed in body tissue, in unit of watt per kilogram $(\mathrm{W} / \mathrm{kg})$ that can be expressed as the following expression (1) [8]:

$$
\mathrm{SAR}=\frac{\sigma}{2 \rho}|E|^{2}
$$

where $\sigma, E$ and $\rho$ are the conductivity of the tissue $(\mathrm{S} / \mathrm{m})$, root mean square $(\mathrm{rms})$ of electric field strength $(\mathrm{V} / \mathrm{m})$, and the mass density of tissue $\left(\mathrm{kg} / \mathrm{m}^{3}\right)$, accordingly. The expression indicates 
that SAR is directly proportional to the conductivity of the tissue and the electric field strength, while inversely proportional to the mass density of the tissue. This SAR measurement used two types of electric field probe known as vector and scalar electric field probes. Vector electric field probe provides phase and magnitude information, while scalar electric field probe gives only magnitude of measured electric field.

Scalar probes are widely implemented in laboratories and reasonably economical compared to the vector probes. Typically, SAR for the single antenna which is implemented in conventional devices is not depending on the phase, but on the magnitude of the measured electric field. Conversely, SAR for the multiple antennas, which work simultaneously at the same frequency is depending on the relative phases of radiating sources as the total electric field is the vector summation of the electric fields from the individual antennas. This issue leads to the biggest challenge as MIMO or phased array antenna systems is the essential structures to cope the demand of convergences for the future 5G technology. According to the global standards established, the International Commission Non-lonizing Radiation Protection (ICNIRP) guideline 1998 [9] fixes $2.0 \mathrm{~W} / \mathrm{kg}$ for $10 \mathrm{~g}$ mass of tissue as a limit of SAR, while American National Standards Institute (ANSI) and Federal Communications Commission (FCC) guidelines and procedures [10] limit $1.6 \mathrm{~W} / \mathrm{kg}$ for $1 \mathrm{~g}$ mass of tissue. Thus to date, the interest of SAR investigation has broadening to the multiple antennas [11]-[14] with concern of interrelation between EMF radiation and human body. In [11] and [12], the SAR investigation focused on two element antennas, which are planar inverted-F antenna (PIFA) at design frequency of $1.9 \mathrm{GHz}$ and textile antenna at $1.5 \mathrm{GHz}$.

The SAR analysis concentrated on different signal weight comprising the phase and magnitude of the input signal. The phase difference of two antennas contributes to major SAR performance. The in-phase signal $\left(0^{\circ}\right.$ and $\left.180^{\circ}\right)$ produces low SAR performance due to the symmetrical distribution of electric field, while the out of phase signal $\left(90^{\circ}\right.$ and $\left.270^{\circ}\right)$ leads to higher SAR value due to unequal spreading of electric field. However, two elements seem to be insufficient number for multiple antennas' SAR investigation. While in [13] and [14], the multiple antennas are considered in 15 and $28 \mathrm{GHz}$ linear array of $1 \times 4$, and $1 \times 8$ configurations and 15 $\mathrm{GHz}$ planar array of $4 \times 4$ configuration for investigation of EMF exposure in term of power density (PD) instead of SAR. The assessment is concentrated on the maximum allowable possible radiated power according to the established basic restriction. However, the SAR investigation of multiple antennas is unexplored at mobile operating frequency between 0.8 to 2.6 GHz. Thus, these [10-13] lead to a sturdy motivation to broaden the SAR investigation over the multiple antennas at mobile operating frequency. As the technology of $5 \mathrm{G}$ stresses the high speed and strong connectivity, hundreds of antennas are predicted to be connected simulataneously to meet the necessary demand. Thus, a significant and attentiveness research on the effect of non-ionizing EMF radiated from these multiple antennas towards human is necessary.

This article addresses the investigation of SAR on single, $1 \times 2,1 \times 3$, and $1 \times 4$ linear antenna arrays of microstrip patch antenna operated at $0.8,0.85,0.9,1.8,2.1$ and $2.6 \mathrm{GHz}$ by utilizing two types of phantoms accessible in CST Microwave Studio 2016, which are specific anthropomorphic mannequin (SAM) and Voxel head model. These frequencies are chosen by concerning the mobile operating frequencies of Global System for Mobile (GSM), Universal Mobile Telecommunications Service (UMTS) and Long Term Evolution (LTE).

\section{Antenna Design}

Initially, a single rectangular microstrip patch antenna is designed by referring to Figure 1. The center frequency of $0.85,1.9$ and $2.6 \mathrm{GHz}$ are chosen to cover the whole respective designated frequencies of $0.8,0.85,0.9,1.8,2.1$ and $2.6 \mathrm{GHz}$. The single antenna has a rectangular-shaped patch on the top layer with length of patch, $L_{p}$ and width of patch, $W_{p}$. While the width of feeding line, $W_{f}$ is designed for $50 \Omega$ to maximizing the power transmission. The substrate is determined comprehensively with length, $L_{s}$ and width, $W_{s}$, which directly affecting the resonance frequency and impedance matching. The substrate length, $L_{s}$ and substrate width, $W_{s}$ are computed from the respective $2 \times L_{p}$ and $1.12 \times W_{p}$. Whilst, the bottom layer consists of rectangular-shaped partial ground plane that have length, $L_{g}$ and width similar to $W_{s}$ as shown in Figure 1 (b). The ground length, $L_{g}$ is fixed to $0.25 L_{s}$. The partial ground plane is considered in this antenna design to overcome a poor reflection performance and 
deficient radiation efficiency from full groud plane due to the excessive input resistance. This partial ground plane introduces an additional reactance, which enhance the radiation efficiency at feeding line and consequently resulting wider performance [15].

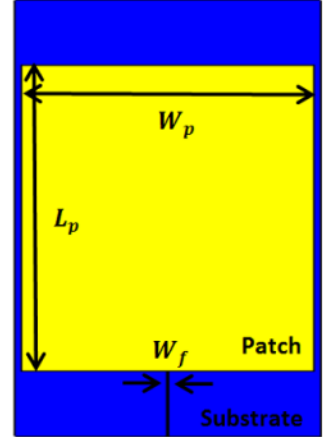

(a)

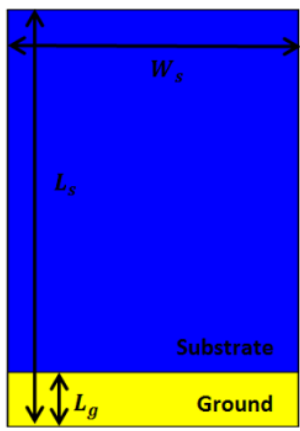

(b)

Figure 1. CST Layout of the Designed Single Microstrip Patch Antenna: (a) Top and (b) Bottom View

Initially, the length, $L_{p}$ and width, $W_{p}$ of the rectangular-shaped microstrip patch antenna can be determined from the respective equations 2 and 3 [16]:

$$
\begin{aligned}
& L_{p}=\frac{c}{2 f_{r} \sqrt{\varepsilon_{e f f}}}-2 \Delta L \\
& W_{p}=\frac{c}{2 f_{r}} \sqrt{\frac{2}{\varepsilon_{r}+1}}
\end{aligned}
$$

where $c, f_{r}, \varepsilon_{e f f}, \varepsilon_{r}$ and $\Delta L$ are the speed of light, designated frequency, the effective dielectric constant, the dielectric constant of the substrate, and the normalized extension in the length due to the fringing effect. The effective dielectric constant, $\varepsilon_{\text {eff }}$ and $\Delta L$ can be computed from (4) and (5), respectively to obtain the optimal performance of antenna [16]:

$$
\begin{aligned}
& \varepsilon_{e f f}=\frac{\varepsilon_{r}+1}{2}+\frac{\varepsilon_{r}-1}{2}\left[1+12 \frac{h}{W_{p}}\right]^{1 / 2} \\
& \Delta L=0.412 h \frac{\left(\varepsilon_{e f f}+0.3\right)\left(\frac{W_{p}}{h}+0.264\right)}{\left(\varepsilon_{e f f}-0.258\right)\left(\frac{W_{p}}{h}+0.8\right)}
\end{aligned}
$$

where $h$ is the thickness of substrate.

Subsequently, the single antenna configuration is applied to design $1 \times 2,1 \times 3$, and $1 \times 4$ antenna arrays with separation distance of half wavelength $(\lambda / 2)$ as shown in Figure 2 . This separation distance offers an optimal mutual coupling between each antenna element. In other cases, such as unequally spaced linear aray, varied separation distances less than wavelength $(\lambda)$ can be considered to be implemented [17]. Several substantial optimizations are conducted in the designs of multiple antennas to achieve the designated frequency ranges. The design applies the high performance of substrate, Rogers RT6010LM that has 10.7 relative permittivity, 0.0023 loss tangent, $18 \mu \mathrm{m}$ conductor coating and $0.254 \mathrm{~mm}$ substrate thickness. This substrate offers size reduction due to its high relative permittivy that suitable for multipleinput multiple-output (MIMO) applications. In addition, this substrate provides wider bandwidth performance due to its good thermal mechanical stability. 


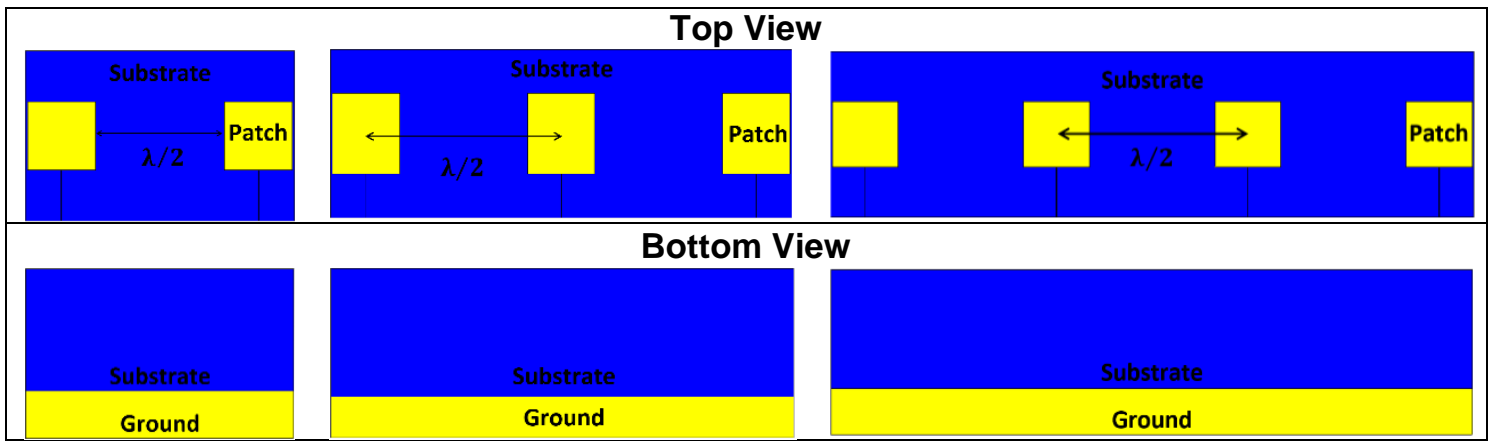

(a)

(b)

(c)

Figure 2. CST Layout of Microstrip Patch Antenna Array from Top and Bottom View: (a) $1 \times 2$ Array, (b) $1 \times 3$ Array and (c) $1 \times 4$ Array

\section{Methodology}

The SAM phantom and Voxel head model are implemented individually with the single, $1 \times 2,1 \times 3$ and $1 \times 4$ arrays of antennas in the investigation of SAR to have comparison of the EMF exposure to the human head tissue. SAM phantom model is representing a control model to provide the range of the highest expected SAR values, while Voxel head model is representing the actual human head with different tissue properties. The built-in SAM phantom model is based on the $90^{\text {th }}$ percentile of male population head size, which consists of a plastic shell filled with a liquid that represents the standardized human head tissue properties [18]. While, the Voxel head model is acquired from one of the models in CST Voxel Family named Gustav, whose is 38 years old male, $163 \mathrm{~cm}$ in size and $51 \mathrm{~kg}$ in mass. This adult head model is employed with the dielectric properties derived from the 4-cole-cole extrapolation for various tissues [19]. The SAR simulation implements SAM phantom and Voxel head model according to the standard averaging procedure defined in the Institute of Electrical and Electronics Engineer / International Electrotechnical Commission (IEEE/IEC 62704-1) standard. This averaging method is evaluated for a cubical volume that contains $10 \%$ of $1 \mathrm{~g}$ or $10 \mathrm{~g}$ of required mass. The single antenna or antenna array is placed at fixed $10 \mathrm{~mm}$ distance from the right ear of SAM phantom or Voxel head model with $19 \mathrm{dBm}(0.08 \mathrm{~W})$ of power excitation as illustrated in the respective Figure 3 and Figure 4.

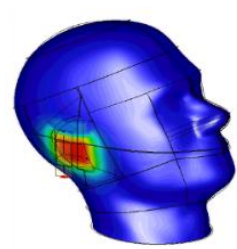

$0.736 \mathrm{~W} / \mathrm{kg}$

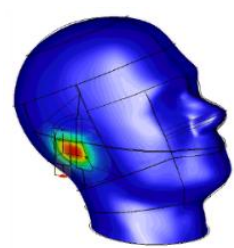

$1.506 \mathrm{~W} / \mathrm{kg}$

(a)

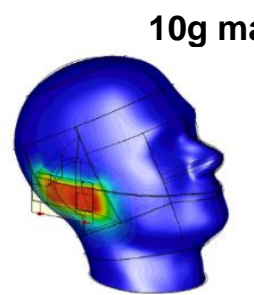

$0.3975 \mathrm{~W} / \mathrm{kg}$

$1 \mathrm{~g}$ mass of tissue

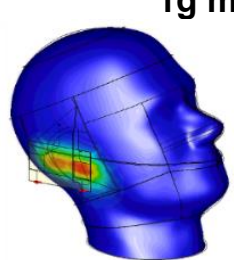

$0.7946 \mathrm{~W} / \mathrm{kg}$

(b)

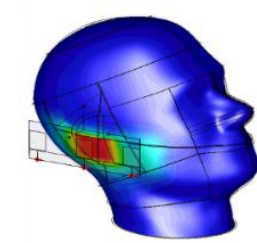

$0.3450 \mathrm{~W} / \mathrm{kg}$

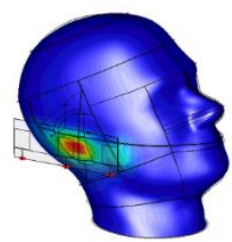

$0.6444 \mathrm{~W} / \mathrm{kg}$

(c)

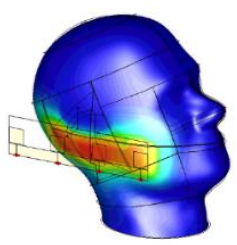

$0.1325 \mathrm{~W} / \mathrm{kg}$

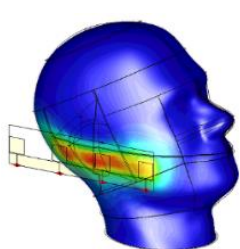

$0.2441 \mathrm{~W} / \mathrm{kg}$

(d)

Figure 3. EMF Radiation and SAR Value for $10 \mathrm{~g}$ and $1 \mathrm{~g}$ Mass of Tissue for (a) Single Antenna, (b) $1 \times 2$ Antenna Array, (c) $1 \times 3$ Antenna Array and (d) $1 \times 4$ Antenna Array at $2.6 \mathrm{GHz}$ that Fixed at $10 \mathrm{~mm}$ Distance from Right Ear of SAM Phantom with Excitation of $19 \mathrm{dBm}(0.08 \mathrm{~W})$. 


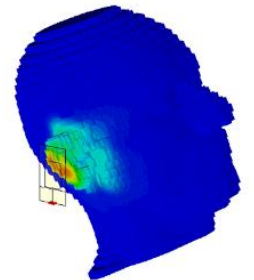

$0.4847 \mathrm{~W} / \mathrm{kg}$

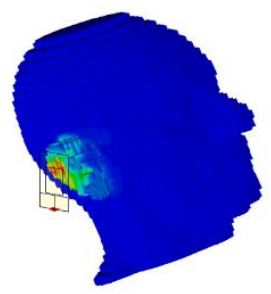

$1.498 \mathrm{~W} / \mathrm{kg}$

(a)

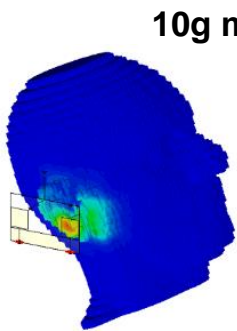

$0.4612 \mathrm{~W} / \mathrm{kg}$

$1 \mathrm{~g}$ mass of tissue

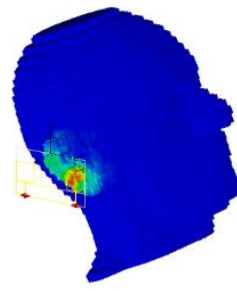

$0.9862 \mathrm{~W} / \mathrm{kg}$

(b)

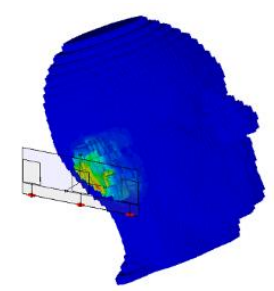

$0.7905 \mathrm{~W} / \mathrm{kg}$

(c)

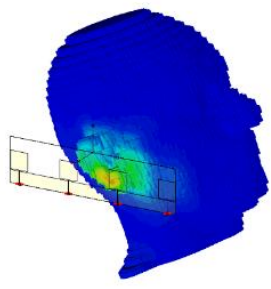

$0.2219 \mathrm{~W} / \mathrm{kg}$

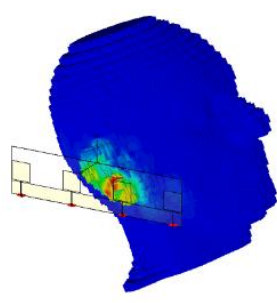

$0.3789 \mathrm{~W} / \mathrm{kg}$

(d)

Figure 4. EMF Radiation and SAR Value for $10 \mathrm{~g}$ and $1 \mathrm{~g}$ Mass of Tissue for (a) Single Antenna, (b) 1 × 2 Antenna Array, (c) $1 \times 3$ Antenna Array and (d) $1 \times 4$ Antenna Array at $2.6 \mathrm{GHz}$ that Fixed at $10 \mathrm{~mm}$ Distance from Right Ear of Voxel Head Model with Excitation of $19 \mathrm{dBm}(0.08$ W).

Distance and excitation power value are kept constant in order to comprehensively study the effect EMF to human tissue. The power excitation is set to the maximum allowable for both SAM and Voxel head model, where the SAR values for the single and multiple antennas are not exceeding the established SAR standard limit for $1 \mathrm{~g}$ and $10 \mathrm{~g}$ tissue over the varied frequencies of $0.8,0.85,0.9,1.8,2.1$ and $2.6 \mathrm{GHz}$. The consistent Hexahedral Fast Perfect Boundry Approximation (FPBA) mesh technology with global number of 10 lines per wavelength are set in the mesh setting [20] to well comparable results throughtout the investigation. Further discussion on the finding will be done in the next Section 4 of Results and Discussions.

\section{Results and Discussions}

The performances of single antenna and multiple antennas are tabulated in Table I in terms of reflection coefficient $\left(S_{11}\right)$, gain, directivity and SAR. While, the trends of single and multiple antennas' SAR performance in bar chart form are depicted in Figure 5. Referring to Table I, the $S_{11}$ performance of the antennas' designated frequencies are performed at lower than $-12.12 \mathrm{~dB}$, which indicates that the antenna has the maximum reflected power of $6.14 \%$. Additionally, the antennas' gain and directivity performance that extracted from the antenna's radiation efficiency indicate the strength of electric field of the antennas. Based on the simulation results obtained, the multiple antennas for each designated frequency offer higher gain value that contributed from the summation of individual antenna gain compared to the single antenna gain. Table I and Figure 5 show that SAR increases with the increment of frequency. However, SAR is decreasing with the increment number of antennas.

\subsection{Electric Field Strength and SAR performance}

The strength of travelling electromagnetic (EM) wave also can be analyzed from the antenna gain for SAR investigation. It is noted that the $1 \times 4$ antenna array at designed frequency of $0.90 \mathrm{GHz}$ provides the highest gain of $9.137 \mathrm{~dB}$. The SAR expression in (1) indicates that SAR value is directly proportional to the electric field strength, which can be obtained from the antenna gain performance. However, this rule of thumb expression of SAR seems only applicable for the single element. From the results obtained, the multiple antennas 
are performed with high electric field strength that might due to the higher gain and directivity performance, which resulting lower SAR. In [21], the SAR values for the multiple antennas is mainly contributed by the arbitrary relative phase combinations as the total field depends on the phase and magnitude of the electric fields from the individual antennas. A significant assumption of only relative phases of the sources are considered as subject to change, whereas the time-averaged value of the magnitude is remaining unchanged at the maximum values of SAR. Referring to Figure 3 and Figure 4 which show the EMF radiaton and the distribution of electric field pattern for designated frequency of $2.6 \mathrm{GHz}$ that fixed at $10 \mathrm{~mm}$ distance from right ear of SAM phantom and Voxel head model with excitation of $19 \mathrm{dBm}(0.08$ $W$ ). The distribution of electric field strength indicated by the red to green colour seems more consistently disperse for multiple antennas compared to single antenna, where the distribution of electric field is intense at certain part of ear. These results may interrelated with one of the indicators in the radition pattern of antenna, known as the $3 \mathrm{~dB}$ beamwidth. This indicator also known as half-power beam width where, the angle in which the relative power is more than $50 \%$ of the peak power is in the effective radiated field of the antenna. The multiple antennas seem to have a smaller $3 \mathrm{~dB}$ beam compared to the single antenna, consequently lower power absorption distributed equivalently by the mass of tissue, which contributed to the lower SAR performance. Thus, the penetration of EMF radiation for the multiple antennas performs a lower SAR value compared to the single antenna. In the other perspective, the trend also shows the increment of SAR over the designated frequency. This is due to the increment of electrical distance to phantom, which implies more superficial absorption as the size of the antenna decrease. Consequently, larger fraction of the absorbed power within the applied SAR averaging volume will contribute to the lower SAR performance.

Table 1. Antenna and SAR Performance

\begin{tabular}{|c|c|c|c|c|c|c|c|c|}
\hline \multirow{2}{*}{ Type } & \multirow{2}{*}{$\begin{array}{c}\text { Frequency } \\
(\mathrm{GHz})\end{array}$} & \multirow{2}{*}{$S_{11}(\mathrm{~dB})$} & \multirow{2}{*}{$\begin{array}{l}\text { Gain } \\
(\mathrm{dBi})\end{array}$} & \multirow{2}{*}{$\begin{array}{l}\text { Directivity } \\
\text { (dB) }\end{array}$} & \multicolumn{2}{|c|}{ SAR for SAM (W/kg) } & \multicolumn{2}{|c|}{ SAR for Voxel (W/kg) } \\
\hline & & & & & $1 \mathrm{~g}$ & $10 \mathrm{~g}$ & $1 \mathrm{~g}$ & $10 \mathrm{~g}$ \\
\hline & 0.80 & -17.09 & 2.114 & 2.429 & 0.287 & 0.214 & 0.1195 & 0.0729 \\
\hline & 0.85 & -36.61 & 2.234 & 2.519 & 0.275 & 0.205 & 0.1133 & 0.0668 \\
\hline Single & 0.90 & -21.22 & 2.342 & 2.618 & 0.260 & 0.194 & 0.1132 & 0.0640 \\
\hline \multirow[t]{5}{*}{ Antenna } & 1.80 & -17.89 & 2.205 & 2.265 & 1.140 & 0.670 & 0.8509 & 0.3804 \\
\hline & 2.10 & -15.75 & 2.394 & 2.503 & 1.232 & 0.679 & 0.9232 & 0.4352 \\
\hline & 2.60 & -38.13 & 2.287 & 2.420 & 1.506 & 0.736 & 1.498 & 0.4847 \\
\hline & 0.80 & -13.42 & 5.165 & 5.373 & 0.1733 & 0.1359 & 0.0781 & 0.0521 \\
\hline & 0.85 & -17.85 & 5.505 & 5.682 & 0.1660 & 0.1301 & 0.0846 & 0.0556 \\
\hline $1 \times 2$ & 0.90 & -17.82 & 5.768 & 5.934 & 0.1572 & 0.1224 & 0.0948 & 0.0563 \\
\hline \multirow[t]{5}{*}{ Array } & 1.80 & -15.05 & 5.152 & 5.391 & 0.4122 & 0.2548 & 0.6089 & 0.2767 \\
\hline & 2.10 & -12.12 & 5.449 & 5.691 & 0.4163 & 0.2516 & 0.6321 & 0.3820 \\
\hline & 2.60 & -18.84 & 5.182 & 5.383 & 0.7946 & 0.3975 & 0.9862 & 0.4612 \\
\hline & 0.80 & -14.39 & 7.380 & 7.667 & 0.0961 & 0.0751 & 0.0664 & 0.0392 \\
\hline & 0.85 & -21.30 & 7.645 & 7.900 & 0.0955 & 0.0751 & 0.0672 & 0.0390 \\
\hline $1 \times 3$ & 0.90 & -17.87 & 7.852 & 8.112 & 0.0951 & 0.0744 & 0.0716 & 0.0401 \\
\hline \multirow[t]{5}{*}{ Array } & 1.80 & -13.95 & 7.643 & 7.719 & 0.4181 & 0.2536 & 0.4411 & 0.2015 \\
\hline & 2.10 & -29.32 & 8.352 & 8.431 & 0.3351 & 0.1920 & 0.3696 & 0.1845 \\
\hline & 2.60 & -19.36 & 7.882 & 7.997 & 0.6444 & 0.3450 & 0.7905 & 0.3149 \\
\hline & 0.80 & -13.68 & 8.576 & 8.757 & 0.0737 & 0.0567 & $96.88 \mu$ & $68.5 \mu$ \\
\hline & 0.85 & -24.97 & 8.889 & 9.038 & 0.0643 & 0.0491 & $0.149 \mathrm{~m}$ & $0.114 \mathrm{~m}$ \\
\hline $1 \times 4$ & 0.90 & -23.77 & 9.137 & 9.271 & 0.0632 & 0.0478 & $0.170 \mathrm{~m}$ & $0.127 \mathrm{~m}$ \\
\hline \multirow[t]{3}{*}{ Array } & 1.80 & -14.49 & 8.538 & 8.778 & 0.1733 & 0.1099 & 0.2144 & 0.1146 \\
\hline & 2.10 & -12.39 & 8.978 & 9.220 & 0.1482 & 0.0916 & 0.2572 & 0.1380 \\
\hline & 2.60 & -28.21 & 8.748 & 8.984 & 0.2441 & 0.1325 & 0.3789 & 0.2219 \\
\hline
\end{tabular}

\subsection{Dielectric Dispersion of Water in Human Tissue and SAR Performance}

From the obtained results, the SAR performance for SAM phantom and Voxel head model show a significant reduction of SAR performance from single antenna to the multiple antenna at each concerned frequencies for both $1 \mathrm{~g}$ and $10 \mathrm{~g}$ mass of tissue. The Voxel head model performs higher SAR value compared to SAM phantom as it contains 45 various types of water-based tissue properties. Where, the dielectric dispersion in biological material plays important role as the dielectric properties are corresponding to the rotational ability of tissue water molecules due to the organic environment and the biological interaction [22]. Thus, the interaction between EMF exposure and dielectric dispersion is dominant, which contributes to 
the higher SAR in Voxel head model. Furthermore, the power absorption is more intense for smaller mass of tissue [23] as expressed in equation 1, where the smaller mass density is more concentrated to the electric field strength.
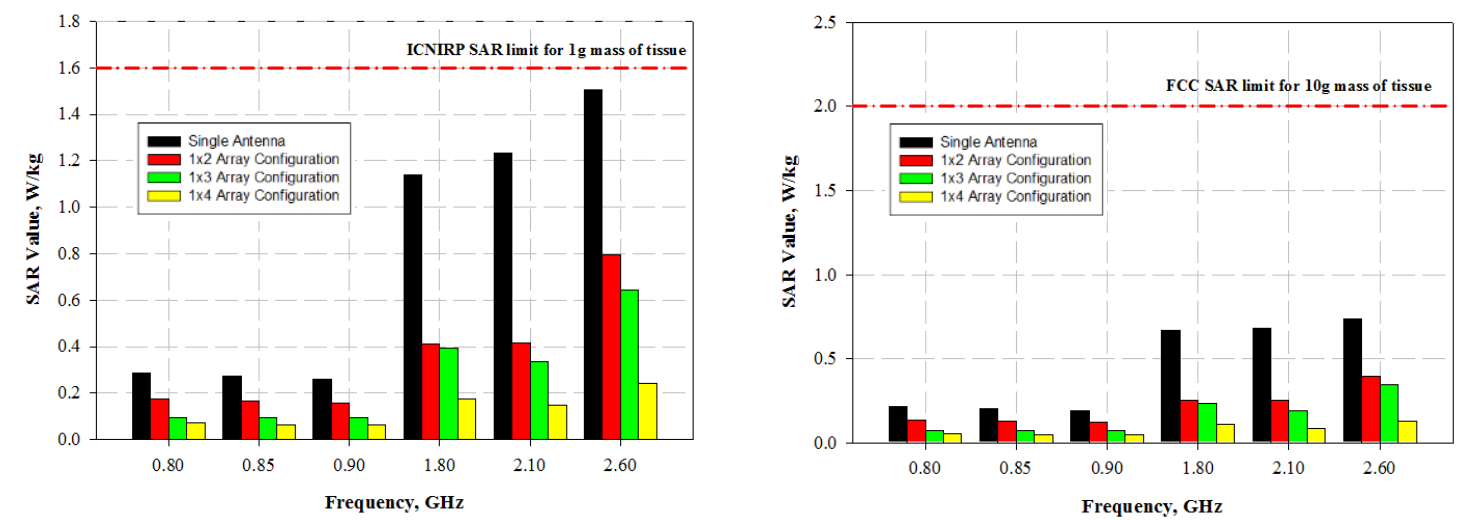

(a)
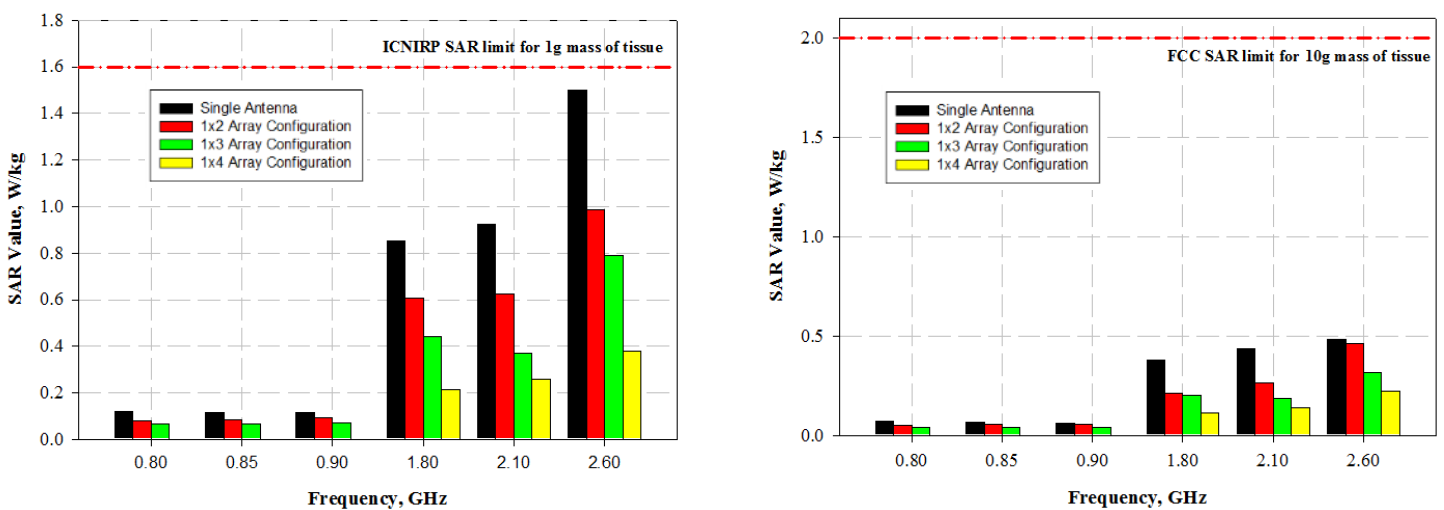

(b)

Figure 5. Single and Multiple Antennas' SAR Trends for $1 \mathrm{~g}$ and $10 \mathrm{~g}$ Mass of Tissue with (a) SAM Phantom, and (b) Voxel Head Model

\section{Conclusion}

Parallel to the aims of $5 G$ technology, this article has focused on multiple antennas, which purposely to enhance gain and the radiation efficiency. The SAR investigation on multiple antennas for designated frequencies of $0.8,0.85,0.9,1.8,2.1$ and $2.6 \mathrm{GHz}$ has been analyzed by applying the SAM phantom and Voxel head model. The relative results for single and multiple antenna have demonstrated the low power absorption performance for multiple antenna in both; SAM phantom and Voxel head model. However, the SAR results over the designated frequencies have shown an increment. This may due to the EMF radiation interaction with the homogenous liquid in SAM phantom and various Voxel head tissue properties. From the results obtained, all designated frequencies for both; single and multiple antennas are below the standard limit established by the ICNIRP and FCC. Therefore, it demonstrates that the multiple antennas are cooperative and inoffensive for the future $5 \mathrm{G}$ technology requirements across the mobile operating frequencies.

\section{References}

[1] J. Sheetal. Architecture of 5G Technology in Mobile Communication. in Proceedings of 18th IRF International Conference. 2015; January: 43-45.

[2] C. X. Wang, F. Haider, X. Gao, X. H. You, Y. Yang, D. Yuan, H. M. Aggoune, H. Haas, S. Fletcher, and E. Hepsaydir. Cellular Architecture and Key Technologies for 5G Wireless Communication N 
etworks. IEEE Commun. Mag. 2014; 52(2): 122-130.

[3] X. Pang, W. Hong, T. Yang, and L. Li. Design and Implementation of an Active Multibeam Antenna System with 64 RF Channels and 256 Antenna Elements for Massive MIMO Application in 5G Wireless Communications. China Commun. 2014; 11(11):16-23.

[4] A. Osseiran, F. Boccardi, V. Braun, K. Kusume, P. Marsch, M. Maternia, O. Queseth, M. Schellmann, H. Schotten, H. Taoka, H. Tullberg, M. a. Uusitalo, B. Timus, and M. Fallgren. Scenarios for 5G Mobile and Wireless Communications: The Vision of the METIS project. IEEE Commun. Mag.. 2014;. 52(5): 26-35.

[5] National Institute of Environmental Health Sciences. Electric \& Magnetic Fields. 2016. [Online]. Available: http://www.niehs.nih.gov/health/topics/agents/emf/. [Accessed: 26-Oct-2016].

[6] J. G. J. G. Andrews, S. Buzzi, W. Choi, S. V. S. V. Hanly, A. Lozano, A. C. K. A. C. K. Soong, and J. C. J. C. Zhang. What will 5 G be?. IEEE J. Sel. Areas Commun. 2014; 32(6):1065-1082.

[7] N. A. Ibrahim, T. A. Rahman, and O. Elijah. Recent Trend in Electromagnetic Radiation and Compliance Assessments for $5 \mathrm{G}$ Communication. International Journal of Electrical and Computer Engineering (IJECE). 2017; 7(2): 912.

[8] H. Li, A. Tsiaras, B. Derat, and B. K. Lau. Analysis of SAR on Flat Phantom for Different MultiAntenna Mobile Terminals. 8th Eur. Conf. Antennas Propagation, EuCAP 2014. 2014; 1: 1989-1993.

[9] J. Herbertz. Guidelines for Limiting Exposure to Time-Varying Electric, Magnetic, and Electromagnetic Fields (Up to $300 \mathrm{GHz}$ ). 1998.

[10] C. Kwok, R. F. Jr. Cleveland, and D. L. Means. Evaluating Compliance with FCC Guidelines for Human Exposure to Radiofrequency Electromagnetic Fields Supplement C. 1997; 65(August): 36.

[11] K.-C. C. K.-C. Chim and R. D. Murch. Investigating the Effect of Smart Antenna on SAR. IEEE Antennas Propag. Soc. Int. Symp. (IEEE Cat. No.02CH37313). 2002; 1: 432-435.

[12] R. Yahya, M. R. Kamarudin, and N. Seman. New Wideband Textile Antenna for SAR Investigation in Head Microwave Imaging. Conf. Proc. - 2014 IEEE MTT-S Int. Microw. Work. Ser. RF Wirel. Technol. Biomed. Healthc. Appl. IMWS-Bio 2014, 2015.

[13] K. Zhao, Z. Ying, S. Member, and S. He. EMF Exposure Study Concerning mmWave Phased Array in Mobile Devices for 5G Communication. IEEE Antennas Wirel. Propag. Lett. 2016; 15: 1132-1135.

[14] D. Colombi, B. Thors, and C. Törnevik. Implications of EMF Exposure Lim vol. 6, no. 1, p. 110.

[18] H. F. Gilbert and M. H. O'Leary. Modification of arginine and lysine in proteins with 2,4-pentanedione. Biochemistry. 1975; 14(23):5194-9.

[19] C. Gabriel. Compilation of the dielectric properties of body tissues at RF and microwave frequencies. Phys. Dep.. 1995; King?s Col(January): 1-13.

[20] N. A. Elias, N. A. Samsuri, M. K. A. Rahim, C. J. Panagamuwa, and W. Whittow. SAR levels for irradiation by a crumpled $900 \mathrm{MHz}$ flexible diamond dipole. International Journal of Electrical and Computer Engineering (IJECE). 2017; 7(3): 1546-1553.

[21] D. T. Le, L. Hamada, S. Watanabe, and T. Onishi. A Fast Estimation Technique for Evaluating the Specific Absorption Rate of Multiple-Antenna Transmitting Devices. 2017; 65(4): 1947-1957.

[22] K. Hosain, F. Samad, and M. S. Alam. Effects of Electromagnetic Fields on Mammalian Cells. International Journal of Electrical and Computer Engineering (IJECE). 2012; 2(2): 267-276,.

[23] M. F. Ali and S. Ray. SAR analysis in a realistic grounded human head for radiating dipole antenna. 2011 Natl. Conf. Commun. NCC 2011. 2011; c: 5-9. 